\title{
Evaluation of diffusion models for airborne nanoparticles transport and dispersion
}

\author{
F. Morency ${ }^{1}, \mathrm{~S}$. Hallé ${ }^{1}$, L. Dufresne ${ }^{1} \&$ C. Émond ${ }^{2}$ \\ ${ }^{1}$ École de technologie supérieure, Département de génie mécanique, \\ Montréal, Canada \\ ${ }^{2}$ Université de Montréal, Département de santé environnementale et \\ santé au travail, Montréal, Canada
}

\begin{abstract}
The diffusion coefficient is a property that plays a significant role in the transport of airborne nanoparticles. However, there seems to be no general agreement in the literature on the most appropriate model to use for nanoparticle numerical simulations to be used in risk exposure assessments. This paper begins by presenting a brief review of some of the main models for small particles diffusion. A general dynamic equation for aerosol transport is briefly discussed next. Since the particle diffusion coefficient can be expressed in terms of a friction coefficient, three relationships are then presented and their influences on the friction and diffusion coefficients are considered for the particular case of $\mathrm{TiO}_{2}$ nanoparticles. Although, all the models studied here predict a decrease in the value of the diffusion coefficient with increasing particle diameter, some significant variations can be observed between the models. A specific diffusion model, chosen between those studied, is finally applied to estimate the purge time of airborne $\mathrm{TiO}_{2}$ nanoparticles in a simple closed space the size of a glovebox. It is shown that the sedimentation and the diffusion processes do not play a major role in the evaluation of the purge time.
\end{abstract}

Keywords: nanoparticles, diffusion, sedimentation, numerical simulations, titanium oxide, purge time.

\section{Introduction}

The evaluation of the potential risks associated with the inhalation of nanoparticles is a question which currently worries many researchers [1]. The 
phenomenal emergence of the nanotechnologies led government agencies to produce discussion papers on the secure use of particles in the nanometric scale. The international reports are unanimous to support proactive measures to ensure the safety of workers exposed to nanoparticles. One of the basic elements for the risk assessment evaluation in a work environment consists of an adequate characterization of the degree of exposure. Computer simulation could be used advantageously as a tool to predict the exposure levels. The development of reliable models will make it possible to predict the behaviour of nanoparticles in a workplace environment, to evaluate the risks of exposure and to contribute to the development of efficient ventilation systems which could make it possible to contain and, if required, to recover the airborne particles. In order to reach theses objectives, numerical models should be able to represent the dominant particles transport and agglomeration mechanisms. The objectives of this paper are to review the present models for small-particle diffusion and to select the most suitable one for nanoparticles transport modelling and risk assessment.

\section{Literature review}

The Brownian diffusion is a phenomenon of passive transport which results from the random movement of the particles subjected to the effect of the collisions with the surrounding molecules. In a macroscopic perspective, the average quadratic displacement of a nanoparticles cause by the Brownian motion can be characterized by a global diffusion coefficient $D$ of the particle [2]. Hinds [2] used the Stokes-Einstein relation to express the diffusion coefficient in term of particule mobility. The resulting expression indicates that the coefficient $D$ is proportional to the temperature of the medium and inversely proportional to the square of the particle diameter $d_{p}$. In spite of this dependence, the diffusion coefficient of a nanoparticle as small as $1 \mathrm{~nm}$ in diameter will be twenty times smaller than the equivalent coefficient for air (molecules). The diffusion of the nanoparticles in air is therefore unlikely to change the trajectory of the nanoparticles significantly compared the main flow streamlines [3]. This "passive scalar" behaviour implies that the nanoparticles can be transported directly by the convective movement of the fluid. However, the diffusion has a determining influence on the "particle-particle" collision probability and on the "particle-wall" collision probability. Therefore the diffusion coefficient may significantly affect the agglomeration of particles between themselves (the coagulation mechanism) or the filter efficiencies.

In a study relating to the theoretical bases of the analytical ultracentrifugation applied to nanoparticles, Lechner and Mächtle [4] make the assumption that the diffusion coefficient is independent of the mass of the particle, inversely proportional to the aerodynamic diameter, and proportional to the absolute temperature. In aerosol science, the aerodynamic diameter of a particle is usually defined as the equivalent diameter of a spherical particle of density equal to $1 \mathrm{~g} / \mathrm{cm}^{3}$ having the same deposit rate or settling velocity as the measured particle [2].

Friedlander [5] proposed a correction factor in order to make the StokesEinstein relationship (see details below) applicable for small particles and large 
mean free paths of the gas molecules. From the results of an analysis based on molecular dynamics, Rudyak et al. [6] developed, for their part, a diffusion model applicable to nanoparticles in dense medium. These latter authors compared their model with the diffusion coefficients obtained from the Einstein [7] and the Enskog kinetic theory of gases [8]. The numerical values of the diffusion coefficients differ considerably according to the model used.

Experimentally, Rudyak et al. [9] compared the results of measurements of the diffusion coefficients with the theoretical predictions of both the Einstein and Enskog theories. Two measuring devices were used for the determination of the $D$ coefficient: a differential mobility analyzer (DMA) and electron microscopy (EM). Measurements obtained from the DMA made use of the Stokes law together with the Cunningham-Millikan-Davis correction. However, this measurement technique led to systematic variations when compared with the theory for very small particles $\left(d_{p}<7 \mathrm{~nm}\right)$. For aerodynamic diameters from 2 to $5 \mathrm{~nm}$, the variations observed between the DMA measurements and the StokesEinstein relation can be as much as several tens of percent. On the other hand, the results obtained from the EM measurements follow very closely the theoretical Stokes-Einstein relation for diameters ranging between 2 and $9 \mathrm{~nm}$. In this same paper, Rudyak et al. also presented a method for the determination of the coefficient $D$ based on the kinetic theory of rarefied gases. This method takes into account the interactions between the surrounding ambient gas molecules and the nanoparticles. The application of this kinetic theory of rarefied gases approach was shown to give diffusion coefficient values between those obtained by DMA and EM measurements.

\section{General dynamic equation}

The transport of nanoscale particles dispersed throughout a fluid is governed by the aerosol general dynamic equation (GDE) [5]. For a discrete distribution function where $k$ refers to the number of molecules in a particle, the GDE is written

$$
\frac{\partial n_{k}}{\partial t}+\nabla \cdot n_{k} \mathbf{v}=\nabla \cdot D \nabla n_{k}+\left[\frac{\partial n_{k}}{\partial t}\right]_{\text {growth }}+\left[\frac{\partial n_{k}}{\partial t}\right]_{\text {coag }}-\nabla \cdot \mathbf{c} n_{k} .
$$

In this equation, $n$ is the concentration, $\mathbf{v}$ is the velocity vector, $D$ is the diffusion coefficient and $\mathbf{c}$ is the particle settling velocity vector resulting from gravity. Coagulation of particles leads to a reduction of the total number of particles, to an increase in the average particle diameter, and to an increase in the number of molecules in the particles. Growth of the particle occurs by gas-toparticle conversion by condensation or nucleation. It increases the particle mass concentration in air.

For particles with large number of molecules, it becomes convenient to pass from a discrete to a continuous distribution to carry out the calculations, but this is outside of the scope of the present paper. In any case, the diffusion coefficient must be determined before solving either the discrete or continuous GDE. The discrete GDE will be solved using classical CFD methods in a future work. 
Classical theory relates the Brownian motion of small particles to the molecular motion of the gas molecules. The Stokes-Einstein expression for the diffusion coefficient is

$$
D=\frac{K T}{f}
$$

where $K$ is the Boltzmann constant and $T$ stands for the absolute temperature. The friction coefficient $f$ furthermore depends on the particle size and on the fluid physical properties.

We will consider here three possible expressions to evaluate this nanoparticle friction coefficient. The appropriate equations depend on the Knudsen number, defined here as twice the ratio of the mean free path of the gas molecules, $l_{p}$, to the particle diameter $d_{p}$ such that

$$
\mathrm{Kn}=\frac{2 l_{p}}{d_{p}} .
$$

For a Kn lower than 1, the Stokes law may be applied. If the particle is considered as being rigid and spherical, the friction coefficient is thus written

$$
f=3 \pi \mu d_{p} \text {. }
$$

For a $\mathrm{Kn}$ much larger than 1 , the friction coefficient can then be determined according to the Enskog kinetic theory $[5,10,11]$ to be

$$
f=\frac{2}{3} d_{p}^{2} \rho\left(\frac{2 \pi k T}{m}\right)^{1 / 2}\left(1+\frac{\pi \alpha}{8}\right)
$$

where $\rho$ is the gas density, $m$ is the molecular mass of the gas molecules, and the accommodation coefficient $\alpha$ is a constant parameter to be taken usually with a numerical value of 0.9 .

A generalization of the above relations can be made to cover a more global range of Kn numbers by rewriting the Stokes law, eqn (4), with a correction factor $C$ such that

$$
f=\frac{3 \pi \mu d_{p}}{C} .
$$

The effects of the Knudsen number are then included in the coefficient $C$, the value of which can in turn be determined by more than one relation. Friedlander [5] and Rudyak et al. [9] suggest the following expression

$$
C=1+\operatorname{Kn}\left(1.257+0.400 \exp \frac{-1.1}{\mathrm{Kn}}\right)
$$

which has been derived from experimental data for oil droplets in air. Hinds [2] proposes a slightly different relation in the form of

$$
C=1+\frac{\mathrm{Kn}}{2}\left(2.34+1.05 \exp \frac{-0.78}{\mathrm{Kn}}\right) \text {. }
$$


Finally, Gussman [12] suggests a relation applicable for different types of gas which reduces to

$$
C=(1+0.615 \mathrm{Kn})^{-1}+1.615 \mathrm{Kn}
$$

for air.

The friction coefficient values predicted by eqns (7)-(9) are shown in figure 1 , for the particular case of $\mathrm{TiO}_{2}$ particles in air at a standard atmospheric pressure of $101.3 \mathrm{kPa}$ and at a temperature of $293 \mathrm{~K}$. Typical $\mathrm{TiO}_{2}$ nanoparticle diameters range from 10 to $100 \mathrm{~nm}$. For reference, values predicted by the kinetic theory in eqn (5) are also plotted. As expected, the kinetic theory tends to over predict the friction coefficient with increasing particle size. Friedlander's eqn (7) and Hinds' eqn (8) give almost the same results and are thus equivalent for these specific conditions.

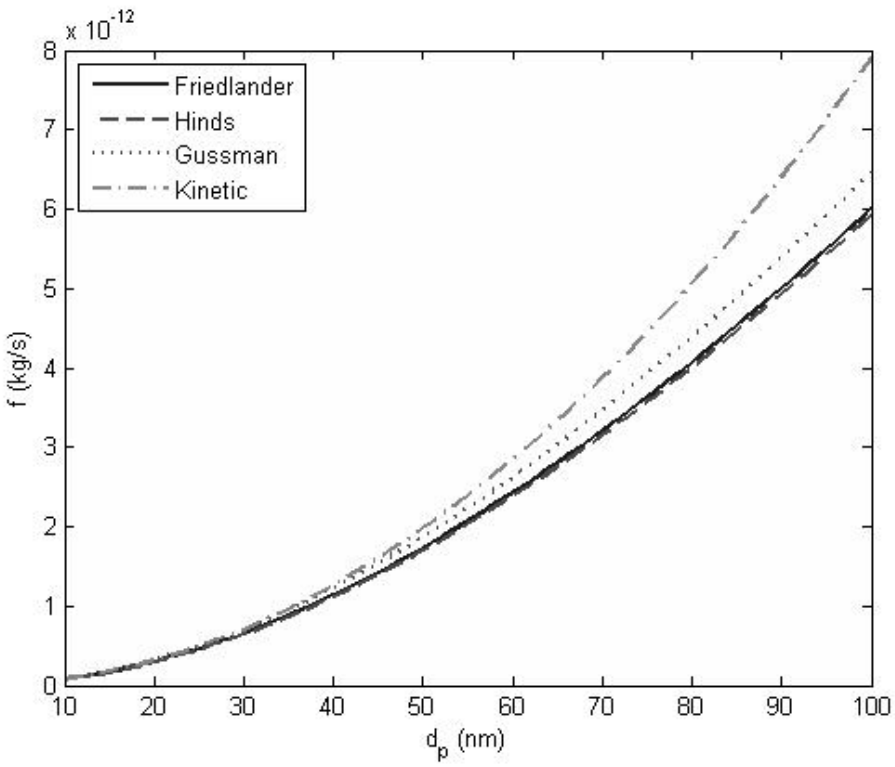

Figure 1: Friction coefficient for $\mathrm{TiO}_{2}$ particle in air at atmospheric pressure.

Gussman's eqn (9) predicts slightly higher values than those of eqn (7) as particle diameter increases, reaching about $8 \%$ difference at $100 \mathrm{~nm}$. On the other hand, for particle diameters below $40 \mathrm{~nm}$, all four relations give very similar results.

As previously mentioned, the numerical values of the diffusion coefficient predicted by eqn (2) depend on the particular expression used to determine the friction coefficient. Figure 2 compares the coefficient $D$ predicted by i) Friedlander's eqn (7), ii) Gussman's eqn (9), iii) the kinetic theory of eqn (5), and iv) the Stokes law in eqn (4). The numerical values shown are obtained for airborne $\mathrm{TiO}_{2}$ particles in air at standard ambient pressure and temperature. As can be seen on the figure, the diffusion coefficients decrease as particle size 


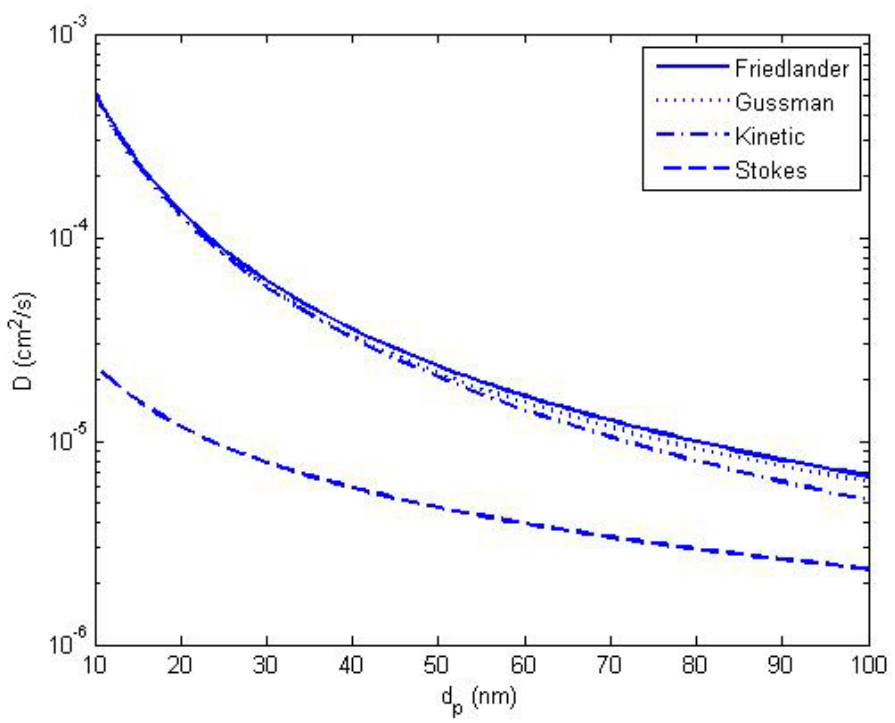

Figure 2: Diffusion coefficient for $\mathrm{TiO}_{2}$ particle in air at atmospheric pressure.

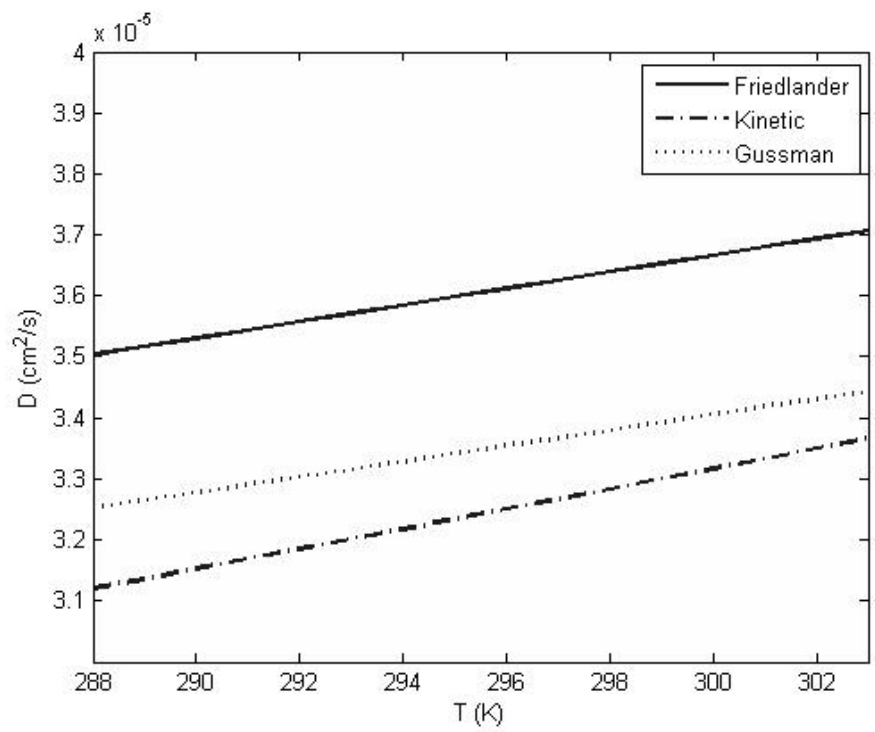

Figure 3: Evolution of the diffusion coefficient with temperature for $40 \mathrm{~nm}$ particles.

increases from $10 \mathrm{~nm}$ to $100 \mathrm{~nm}$. As expected, the Stokes law under predicts the values of $D$. For particle diameters greater than about $40 \mathrm{~nm}$ (as for $f$ ), the kinetic 
theory gives results different from those obtained from Friedlander's and Gussman's equations, which on the other hand agree quite well between themselves. Note that Hinds' equation results are not shown, as they are almost identical to those of Friedlander.

In figure 3, we show the effect of temperature on the diffusion. The curves are obtained for $40 \mathrm{~nm}$ particles in air, again at standard atmospheric pressure. The temperature value range is chosen to cover normal room conditions. The variation of the air viscosity in Stokes eqn is taking into account using Sutherland's correlation [2]. The three diffusion curves show once more the same trend, a nearly linear increase of the diffusion coefficient with temperature, a somewhat similar feature as for general gas behaviour. Friedlander's, the kinetic theory, and Gussman's equation results have about the same slope of a $5 \%$ augmentation for a $15 \mathrm{~K}$ temperature increase.

\section{Particle extraction from a small room}

In the eventuality of an airborne, possibly toxic, event involving nanoparticles in a workplace, it will certainly be critical to be able to estimate the time to completely purge the contaminated air from the room in which it took place, i.e., to remove as much as possible of the unwanted particles from that room. A first estimate of this purge time can be obtained knowing the volume of the room which could then divided by the volume flow rate $Q$ of fresh air from the ventilation system. The time scale hence obtained represents the time taken by the ventilation system to achieve a single volume air exchange.

It may be important to note that for airborne nanoparticles, the diffusion effects close to the walls of the room and the sedimentation process may have a non negligible influence on the evolution in time of the particle concentration in that room. In order to take into account these additional effects, Hervé-Bazin [13] suggests the following exponential relation for the evolution of particle concentration $C$ with time

$$
C=C_{o} \cdot \exp (-t / T)
$$

where $C_{o}$ is the initial concentration, and $T$ a constant time scale such that

$$
\frac{1}{T}=\frac{Q}{V}+\beta
$$

with $\beta$ representing the deposition coefficient in turn obtained by the following relation

$$
\beta=\frac{V_{s}}{h}+\frac{S \cdot D}{V \cdot \delta} .
$$

In the last relation, eqn (12), $V_{S}$ is the sedimentation or settling velocity of the particles, $h$ is the room height, $S$ is the room total wall surface, $V$ is the room volume, and finally $\delta$ an estimate of the boundary layer thickness formed at the surface of the room's wall. 
To give an idea of the purge time of a room, consider a set of $\mathrm{TiO}_{2}$ nanoparticles uniformly distributed in a room with an initial, fairly low, concentration of $10^{4}$ particles per cubic centimetre; at this concentration level, particle coagulation or agglomeration effects can reasonably be neglected [2]. If we now consider a rectangular closed space having the dimensions of a typical glovebox which could be used for nanoparticle experimentations, that is with a $1 \mathrm{~m}$ length, a $0.8 \mathrm{~m}$ width, and a $0.8 \mathrm{~m}$ height. The sedimentation velocity $V_{S}$, following Friedlander's general notation [5], depends on the particle density $\rho_{p}$, the air density $\rho$, and gravity $g$ such that

$$
V_{s}=\frac{\rho_{P} \cdot g \cdot d_{p}^{2}}{18 \mu} C\left(1-\rho / \rho_{p}\right) .
$$

For $\mathrm{TiO}_{2}$ particles $\left(\rho_{p}=4 \mathrm{~g} / \mathrm{cm}^{3}\right)$ in air, one may readily note that the particle's buoyancy term, $\left(1-\rho / \rho_{p}\right)$ is negligibly close to 1 and could thus be omitted.

In table 1 , we show values of $D, V_{S}, \beta$, and $T$, for typical $\mathrm{TiO}_{2}$ particles of 10 $\mathrm{nm}, 20 \mathrm{~nm}, 40 \mathrm{~nm}$ and $100 \mathrm{~nm}$ diameters. These results have been obtained assuming an air flow rate of $Q=4.5 \times 10^{-3} \mathrm{~m}^{3} / \mathrm{s}$ whence $Q / V=7.0 \times 10^{-3} 1 / \mathrm{s}$. in eqn (11). Based on the quasi one dimensional (piston) flow hypothesis made above, we may estimate the particle purge time of the glovebox, $T$, to be at slightly more than $20 \mathrm{~s}$. More specifically, for the smallest particles $(10 \mathrm{~nm})$ sedimentation effects are not significant and the purge time is thus the longest at $26.3 \mathrm{~s}$. For $20 \mathrm{~nm}$ particles, Brownian motion and sedimentation are maximum hence giving the minimal time of $22.4 \mathrm{~s}$. For 40 and $100 \mathrm{~nm}$, the sedimentation velocity increases but the Brownian motion diminishes giving in this case purge time values a bit higher than for the $20 \mathrm{~nm}$ particles but still below the $10 \mathrm{~nm}$ value. One may also deduce from the table results that the sedimentation time scale $h / V_{S}$ is significantly much larger (at least 4 orders of magnitude) than the global purge time $T$, confirming thus that the sedimentation or settling process plays a negligible role in the evolution of the particle concentration.

Table 1: $\quad$ Characteristics of $\mathrm{TiO}_{2}$ particles dispersion in a rectangular closed space (see text for details).

\begin{tabular}{|r||c|c|c|c|}
\hline$d_{p}[\mathrm{~nm}]$ & $D\left[\mathrm{~cm}^{2} / \mathrm{s}\right]$ & $V_{S}[\mathrm{~cm} / \mathrm{s}]$ & $\beta[1 / \mathrm{s}]$ & $T[\mathrm{~s}]$ \\
\hline \hline 10 & $5.2 \mathrm{e}-4$ & $2.6 \mathrm{e}-5$ & $3.7 \mathrm{e}-5$ & 26.3 \\
\hline 20 & $1.3 \mathrm{e}-4$ & $5.4 \mathrm{e}-5$ & $1.0 \mathrm{e}-5$ & 22.4 \\
\hline 40 & $3.5 \mathrm{e}-5$ & $1.2 \mathrm{e}-4$ & $3.9 \mathrm{e}-6$ & 22.5 \\
\hline 100 & $6.7 \mathrm{e}-6$ & $3.4 \mathrm{e}-4$ & $4.7 \mathrm{e}-6$ & 22.7 \\
\hline
\end{tabular}

\section{Conclusion}

The present paper is part of an ongoing work on the development of a numerical simulation model for the dispersion of airborne nanoparticles to be used in risk 
exposure assessments. Our main concern at this stage has been on the evaluation of already existing diffusion models. More specifically, three models have been studied. These models are based on a generalization of the Stokes law for the dynamics of small spherical particles to which a correction factor is added to take into account the very small scale effects occurring at nanoscales. At given standard pressure and temperature, the diffusion coefficient is shown to decrease as the particle diameter increases from 10 to $100 \mathrm{~nm}$. The diffusion models proposed by Friedlander and Hinds give almost identical results while the model proposed by Gussman gives slightly lower values. For particle diameters below $40 \mathrm{~nm}$, the difference between the three models becomes very small though. All models are also shown to agree with the results obtained using a more classical kinetic theory approach for small particle diameters, but that latter theory tends to under predict diffusion for larger diameters. For a given particle diameter, diffusion is shown to increase with temperature, as it is the case in general for gases.

The Friedlander diffusion model was then applied to the evaluation of the time taken to extract a low-level concentration of $\mathrm{TiO}_{2}$ nanoparticles from a small closed room the size of a glovebox. Assuming a certain air flow rate, we were then able to estimate a room purge time. From the values hence obtained, it was then shown that the sedimentation or settling of nanoparticles is negligible in the global dispersion process.

Future work includes the implementation of this diffusion model to more complete fluid dynamics simulation tools in order to be able to obtain more accurate estimations of the purge time in complex room geometries. The inclusion of particle agglomeration or coagulation dynamics will also be considered in order to be able to take higher and more realistic concentration of nanoparticles into account.

\section{References}

[1] Dawson, J., Nanotech risk research takes slow step forward, Phys. Today, 60(11), pp. 29-30, 2007.

[2] Hinds, W. C., Aerosol Technology: Properties, Behavior, and Measurement of Airborne Particles, John Wiley \& Sons, New York, 1999.

[3] Maynard, A.D. \& Kuempel, E.D., Airborne nanostructured particles and occupational health, J. Nanopart. Res. 7, pp. 587-614, 2005.

[4] Lechner, M.D. \& Mächtle, W., Determination of the particle size distribution of 5-100 $\mathrm{nm}$ nanoparticles with the analytical ultracentrifuge: consideration and correction of diffusion effects. Progr. Colloid. Polym. Sci. 113, pp. 37-43, 1999.

[5] Friedlander, S. K., Smoke, Dust, and Haze. Fundamentals of Aerosol Dynamics, Oxford Univ. Press, New York, 2000.

[6] Rudyak, V.Ya., Kharlamov, G.V. \& Belkin, A.A., Diffusion of nanoparticles and macromolecules in dense gases and liquids. High Temp., 39(2), pp. 264-271, 2001.

[7] Einstein, A., Investigation on the theory of Brownian movement, R. Furth editor, Dover Publ., New-York, 1956. 
[8] Ferziger, J.H. \& Kaper, H.G., Mathematical theory of transport in gases. Amsterdam, North-Holland, 1972.

[9] Rudyak, V. Y., Krasnolutskii, S. L., Nasibulin, A.G. \& Kauppinen, E.I., Methods of Measuring the Diffusion Coefficient and Sizes of Nanoparticles in a Rarefied Gas. Dokl. Phys., 47(10), pp. 758-761, 2002.

[10] Settumba, N. \& Garrick, S. C., A comparison of diffusive transport in a moment method for nanoparticle coagulation. Aerosol Sci. 35, pp. 93-101, 2004.

[11] Chan, T. L., Lin, J. Z., Zhou, K. \& Chan, C.K., Simultaneous numerical simulation of nano and fine particle coagulation and dispersion in a round jet. Aerosol Sci. 37, pp. 1545-1561, 2006.

[12] Gussman, R. A., On the Aerosol Particle Slip Correction Factor. J. Appl. Meteorol., 8, pp. 999-1001, 1969.

[13] Hervé-Bazin, B., Les nanoparticules: Un enjeu majeur pour la santé au travail, E.D.P. Sciences, Paris, 2007. 